\title{
Quantum walks in optical lattices
}

\author{
W. Dür, ${ }^{1}$ R. Raussendorf, ${ }^{1}$ V. M. Kendon, ${ }^{2}$ and H.-J. Briegel ${ }^{1}$ \\ ${ }^{1}$ Sektion Physik, Ludwig-Maximilians-Universität München, Theresienstrasse 37, D-80333 München, Germany \\ ${ }^{2}$ Optics Section, Blackett Laboratory, Imperial College, London SW7 2BW, United Kingdom
}

(Received 24 July 2002; published 22 November 2002)

\begin{abstract}
We propose an experimental realization of discrete quantum walks using neutral atoms trapped in optical lattices. The quantum walk is taking place in position space and experimental implementation with present-day technology—even using existing setups—seems feasible. We analyze the influence of possible imperfections in the experiment and investigate the transition from a quantum walk to the classical random walk for increasing errors and decoherence.
\end{abstract}

DOI: 10.1103/PhysRevA.66.052319

PACS number(s): 03.67.Lx, 32.80.Pj

\section{INTRODUCTION}

The increasing effort to investigate theoretically and experimentally the possibility to construct and build a universal quantum computer is mainly motivated by the expectation that quantum computers offer a (possibly exponential) speedup over classical computers. Despite the two celebrated milestones of Shor's factoring algorithm [1] and Grover's database search algorithm [2] — which both offer a speedup over their best (known) classical counterpart—no constructive way to generate efficient quantum algorithms is currently known. One possible direction of research is the adaption of known classical algorithms to the quantummechanical case.

Random walks on graphs play an essential role in various fields of natural science, ranging from astronomy, solid-state physics, polymer chemistry, and biology to mathematics and computer science [3]. In particular, Markov chain simulation has emerged as a powerful algorithmic tool and many classical algorithms, such as approximating the permanent, graph connectivity or $k$ SAT [4] are based on random walks. It is possible and hoped that quantum walks allow in a similar way, a constructive search for new quantum algorithms. This justifies the increasing effort in the investigation of quantum walks by several groups [5-15]. Different behavior of the quantum walk-as compared to the classical random walkhave been reported under various circumstances. For instance, a very promising feature of a quantum walk on a hypercube, namely an exponentially faster hitting time as compared to a classical random walk, has been very recently found (numerically) by Yamasaki et al. [12] and (analytically) by Kempe [11]. Indeed, first quantum algorithms based on quantum walks which offer an (exponential) speedup over their optimal classical counterpart have been reported in Refs. [34,35].

In this paper, we consider the simplest and best-studied version of a quantum walk, namely the discrete Hadamard walk on a line or a circle, first studied in Ref. [5]. We propose an experimental implementation of the quantum walk using neutral atoms trapped in an optical lattice. In contrast to the recently proposed implementations using ion traps put forward by Travaglione and Milburn [13] and microwave cavities put forward by Sanders et al. [15], in our proposal the quantum walk is taking place in position space and sev- eral hundred steps may be implementable even with the present-day technology.

The paper is organized as follows. In Sec. II, we compare features of the classical and quantum walks on the line and introduce some basic notation. Section III provides a description of the physical setup using optical lattices and implementations of the Hadamard walk on a line using this setup. Possible imperfections and their influence on the quantum features of the walk are discussed in Sec. IV. We summarize and conclude in Sec. V.

\section{CLASSICAL WALK VS QUANTUM WALK}

\section{A. Classical random walk on a line}

Consider an infinite line with allowed (integer) positions $x_{k} \equiv k, k \in \mathbb{Z}$ and a particle which is initially located at position $x_{0}=0$. We consider a stepwise evolution in such a way that at each step, the particle moves with probability $1 / 2$ one step to the left, $x(n)=x(n-1)-1$, and with probability $1 / 2$ one step to the right, $x(n)=x(n-1)+1$. After $n$ steps, the probability $p_{\text {classical }}(n, k)$ to find the particle at position $x_{k}$ is given by

$$
p_{\text {classical }}(n, k)=\frac{1}{2^{n}}\left(\begin{array}{c}
n \\
\frac{k+n}{2}
\end{array}\right) .
$$

Note that if $n$ is even (odd), only even (odd) positions are occupied. The standard deviation of the distribution is $\sqrt{n}$, which implies a spreading time proportional to $\sqrt{n}$. The probability to observe a particle at a distance of order $n$ from the origin decreases exponentially with $n$ and is zero $\forall n_{0}$, $n_{0}>n$.

\section{B. Quantum walk on a line}

A quantum-mechanical analogy for the classical random walk would be a particle, whose state evolves at each step into a coherent superposition of moving one step to the right and one step to the left. One readily finds [16] that unitarity of the evolution implies that one has to consider a particle with internal degrees of freedom to achieve this aim. We consider a particle with two internal degrees of freedom, which can move on an infinite line with integer positions $[7,8]$. The corresponding Hilbert space $\mathcal{H}=\mathcal{H}_{I} \otimes \mathcal{H}_{X}$ is given by $\mathcal{H}_{I}=\mathrm{C}^{2}$, the internal state of the particle, and $\mathcal{H}_{X}=\mathrm{C}^{\infty}$, the 
position space with basis states $|k\rangle_{X} \in \mathcal{H}_{X},-\infty \leqslant k \leqslant \infty$, corresponding to the particle located at the $k^{\text {th }}$ lattice site.

The internal state of the particle, $|0\rangle,|1\rangle$ determines the direction of the particle movement. If the internal state is $|0\rangle$, the particle moves to the left, while it moves to the right if the internal state is $|1\rangle$. This operation is described by the unitary controlled-shift operation,

$$
S=|0\rangle\left\langle 0\left|\otimes \sum_{k}\right| k-1\right\rangle\langle k|+| 1\rangle\left\langle 1\left|\otimes \sum_{k}\right| k+1\right\rangle\langle k|,
$$

i.e., $S|0\rangle_{I} \otimes|k\rangle_{X}=|0\rangle_{I} \otimes|k-1\rangle_{X}$ and $S|1\rangle_{I} \otimes|k\rangle_{X}=|1\rangle_{I} \otimes \mid k$ $+1\rangle_{X}$

We also introduce the Hadamard operation

$$
H=\frac{1}{\sqrt{2}}\left(\begin{array}{cc}
1 & 1 \\
1 & -1
\end{array}\right),
$$

which acts on Hilbert space $\mathcal{H}_{I}$ such that $H|0\rangle=1 / \sqrt{2}(|0\rangle$ $+|1\rangle)$ and $H|1\rangle=1 / \sqrt{2}(|0\rangle-|1\rangle)$. The particle is initially prepared in state $\left|\psi_{0}\right\rangle=1 / \sqrt{2}\left(|0\rangle_{I}+i|1\rangle_{I}\right) \otimes|0\rangle_{X}$ [17]. Each step of the quantum walk-which is also called Hadamard walk-consists of applying the Hadamard operation, $H \otimes 1$, followed by the controlled-shift operation $S$. Let $\left|\psi_{n}\right\rangle$ $=(S H)^{n}\left|\psi_{0}\right\rangle$ be the state of the system after $n$ steps. The probability $p(n, k)$ to observe a particle at position $k$ after $n$ steps is given by

$$
p(n, k)=\operatorname{tr}_{X}\left[|k\rangle_{X}\langle k| \operatorname{tr}_{I}\left(\left|\psi_{n}\right\rangle\left\langle\psi_{n}\right|\right)\right]
$$

and may be compared to the probability distribution $p_{\text {classical }}(n)$ of the classical random walk. The probability distribution $p(n)$ has been analyzed in detail in Ref. [8]. While $p_{\text {classical }}(n)$ is given by a binomial distribution - which is for large $n$ well approximated by a Gaussian $-p(n)$ differs greatly from a binomial distribution. The standard deviation of the distribution $p_{\text {classical }}(n)$ is $\sqrt{n}$, while $p(n)$ is almost uniformly distributed in the interval $(-n / \sqrt{2}, n / \sqrt{2})$ and the standard deviation is linear in $n$. This implies that the spreading time for a particle goes like $\sqrt{n}$ for the classical random walk, while in the quantum walk it scales linearly with $n$. This provides an essential different behavior of the quantum walk that follows from the possibility of interference in the quantum-mechanical case.

In a similar way, the (quantum) walk on a circle is defined using a position space $\mathcal{H}_{X}=\mathrm{C}^{N}$ with periodic boundary conditions, i.e., $|k\rangle_{X}=|k \bmod (N)\rangle_{X}$ for some finite $N$. Also in this case, a quadratic speedup of the quantum walk compared to the classical random walk is found in the spreading time of the particle [7]. Walks on general graphs can be defined in a similar way [7].

\section{IMPLEMENTATION IN OPTICAL LATTICES}

In this section, we discuss possible implementations of the quantum (Hadamard) walk on a line or on a circle using neutral atoms trapped in periodic optical potentials (for a review see, e.g., Refs. [18,19]).

\section{A. Physical setup}

We consider two identical one-dimensional (1D) optical lattices, each of them trapping one of the internal states $|0\rangle,|1\rangle$ of a neutral atom. For example, one may use alkali atoms with a nuclear spin equal to $3 / 2\left({ }^{87} \mathrm{Rb},{ }^{23} \mathrm{Na}\right)$, and choose the hyperfine structure states $\left|F=1, m_{f}=1\right\rangle[\mid F$ $\left.\left.=2, m_{f}=2\right\rangle\right]$ to represent $|0\rangle[|1\rangle]$, respectively.

Each lattice consists of a periodic optical potential with period $d$. The optical potentials are formed by the standing waves resulting from two counterpropagating traveling waves with the electric fields forming an angle of $2 \theta$, the so-called lin $\angle$ lin configuration. By changing $\theta$, the right and left circular polarized components $\sigma^{ \pm}$of the standing waves forming the total electric field can be shifted with respect to each other, $\vec{E}^{+}(x, t)=E_{0} e^{-i \nu t}\left[\vec{\epsilon}_{+} \sin (k x+\theta)+\vec{\epsilon}_{-} \sin (k x-\theta)\right]$. We have denoted $k=\nu / c$ as the laser wave vector, $E_{0}$ as the amplitude, and $\vec{\epsilon}_{ \pm}$as unit right and left circular polarized vectors. The potentials "seen" by the atoms in the internal states $|0\rangle,|1\rangle$ are $V_{0}(x, \theta)=\left[V_{m_{s}=1 / 2}(x, \theta)\right.$ $\left.+3 V_{m_{s}}=-1 / 2(x, \theta)\right] / 4$ and $V_{1}(x, \theta)=V_{m_{s}=1 / 2}(x, \theta)$, where $V_{m_{s}= \pm 1 / 2}(x, \theta)=\alpha\left|E_{0}\right|^{2} \sin ^{2}(k x \pm \theta)[20]$.

This basic architecture can be used for quantum state control of neutral atoms in optical lattices [18] and it constitutes the basis of the proposals for quantum computation in such systems [20,21]. As in the proposal of Ref. [20], we make use of the fact that a relative movement of the two lattices, i.e., the trapping potentials $V_{0}, V_{1}$ can be achieved by varying the angle $\theta$. In particular, starting with $\theta=0$, the respective minima of the potentials $V_{0}, V_{1}$ coincide and by changing $\theta$ from 0 to $\pi / 2$, the potentials $V_{0}, V_{1}$ move in opposite directions until their respective minima coincide again. Note that the shape of the potential $V_{0}$ changes as it moves.

\section{B. Implementation of the Hadamard walk}

We consider a single neutral atom at position $x_{0}=0$ and the case where lattice 0 - which traps the internal state $|0\rangle$ of the neutral atom-moves with constant velocity to the left, $v_{0}=-v$, while lattice $1-$ which traps the internal state $|1\rangle$ of the atom-moves with a constant velocity $v_{1}=v$ to the right. The initial position of the lattices is such that the minimum of a potential well is located at position $x_{0}$ at $t_{0}=0$. The lattice movements are used to implement the controlled-shift operation [see Eq. (2)], while laser pulses allow one to manipulate the internal state of the atom, and thus to select the corresponding trapping potential (and therefore the direction of the movement).

Given that the atom is initially prepared in state $1 / \sqrt{2}(|0\rangle+i|1\rangle)[17]$ at position $x_{0}=0$, the application of the Hadamard operation [see Eq. (3)] to the internal state of the atom at times $t_{n}=n d / v$ readily implements the quantum walk on a line using this setup. The spatial probability distribution of the atom at time $t_{n}$, i.e., the probability to observe an atom at position $k d,-n \leqslant k \leqslant n$ at time $t_{n}$ corresponds exactly to $p(n, k)[\mathrm{Eq}$. (4)] of the one-dimensional Hadamard walk after $n$ steps. A simple fluorescence measurement, together with several repetitions of the experiment-allows one to measure this distribution. 
To justify this statement, note that a single atom initially at position $x_{0}=0$, which is prepared in state $|0\rangle[|1\rangle]$ moves with constant velocity to the left, $x(t)=x_{0}-v t$ [right, $x(t)$ $\left.=x_{0}+v t\right]$, respectively. After a time $t_{n}=n d / v$, the position of an atom is shifted by exactly $n$ lattice periods $n d$ and the two lattices are again on top of each other. By changing the internal state of the atom, e.g., at time $t_{n}$ from $|0\rangle$ to $|1\rangle$, one can switch between the corresponding trapping potential and thus change the direction of the movement. Note that it is important to make such changes in the internal state of the atom only when the two lattices are on top of each other, to ensure that the atom remains trapped in one of the potentials. Coherent superpositions of two internal states behave likewise. In the case of Rubidium with $|0\rangle=\left|F=1, m_{f}=1\right\rangle$, $|1\rangle=\left|F=2, m_{f}=2\right\rangle$, one can use standard Raman pulse or microwave techniques to realize the Hadamard rotation by using fast laser pulses.

Note that on the Blochs sphere, the Hadamard operation corresponds to a rotation of an angle $\pi$ around the axis $\vec{u}$ $=1 \sqrt{2}\left(\overrightarrow{e_{x}}+\overrightarrow{e_{z}}\right)$. This corresponds to a $\pi$ pulse rather than a $\pi / 2$ pulse in the usual terminology of quantum optics. Up to an irrelevant global phase, one may also achieve the Hadamard operation by a sequence of three $\pi / 2$ pulses, $H$ $\propto e^{-i \pi / 4 \sigma_{x}} e^{-i \pi / 4 \sigma_{y}} e^{-i \pi / 4 \sigma_{z}}$. Experimentally, it may be easier to use a $\pi / 2$ pulse corresponding to the transformation $U_{\pi / 2}=e^{-i \pi / 4 \sigma_{x}}$ instead of the Hadamard operation, and prepare the atom initially in state $1 / \sqrt{2}(|0\rangle+|1\rangle)$. This also leads to a symmetric probability distribution for all times $t$ equivalent to the one resulting from the standard Hadamard walk.

The Hadamard operation has to be applied at all lattice sites, which can be easily achieved by using a nonfocused laser beam. In fact, such a homogeneous operation $H^{\otimes N}$ is much easier to implement than individual operations on specific lattice sites. This is due to the fact that in current experiments, the lattice period $d \approx 425 \mathrm{~nm}$-which is limited by the optical wavelength-is smaller than the best achievable focusing width of the laser beams, $w \approx 1 \mu \mathrm{m}$ [25]. In the fluorescence measurement, one can either detect unselectively both internal states $|0\rangle$ and $|1\rangle$ to reveal information about the position of the atom, or one may use selective resonance fluorescence methods. In the latter case, addition application of a random $\sigma_{x}$ operation ( $\pi$ pulse) before the measurement is required to remove the dependence on the internal state of the spatial probability distribution. Provided the atom was initially prepared in state $1 / \sqrt{2}(|0\rangle+i|1\rangle)$, the probability distribution is symmetric when tracing out the internal state of the atom, however the distributions conditioned on the internal state of the atom are asymmetric and have mirror symmetry, which explains the additional application of a random $\sigma_{x}$ operation. Notice the phase $i$ in the initial state, which is important to ensure symmetric behavior of the quantum walk.

We would like to emphasize that the procedure sketched above is readily implementable with existing technology. It does not require addressability of individual lattice sites.

The essential requirements are that the internal states of the atom-as well as their coherences-are sufficiently stable and that the particle remains trapped in the potential throughout the procedure. This can be satisfied if the movement of the lattice is sufficiently slow (that is $v \ll \nu_{\text {osc }}$, where $\nu_{\mathrm{osc}} \approx a_{0} \omega$ is the rms velocity of the atoms in the vibrational ground state, $\omega$ is the excitation frequency, and $a_{0}$ is the size of the ground state of the trap potential [20]) such that the atom stays in the ground state of the potential during motion. This condition can be relaxed, as will be discussed in the subsequent section, and one can also allow nonadiabatic velocity profiles. The coherence of the internal state is mainly affected by fluctuations, the intensity and phase of the trapping lasers [26] as well as magnetic-field fluctuations, which may lead to uncontrollable energy splittings between the internal levels. We will address some of these issues in the following section. Given that these noise sources can be controlled sufficiently well, the number of steps of the quantum walk one can perform is only limited by the spontaneous emission lifetime of the atom in the lattice, which is of the order of several seconds. This corresponds to a maximum number of about $n=10^{4}$ time steps, assuming $t_{1}$ $\approx 100 \mu \mathrm{s}--1 \mathrm{~ms}$, which respects the adiabaticity requirement for lattice shifts. Note that the implementation of several hundred time steps of the Hadamard walk corresponds to a spatial width of the quantum distribution at the order of millimeters.

\section{Improved implementation of the Hadamard walk}

From a practical point of view, there are a number of difficulties with the procedure proposed in Sec. III B. For example, the laser pulses to implement the Hadamard rotation have to be fast compared to the time scale of the lattice movement. In addition, if the internal state of an atom is changed, e.g., from $|0\rangle$ to $|1\rangle$, this implies a sudden momentum change of the atom, as it is no longer trapped in the left-moving lattice but in the right-moving one. This momentum change may lead to heating of the atom and the atom may eventually even escape from the trap.

Another practical difficulty, one faces in current experiments is concerned with dephasing of the internal states of the atom. In particular, uncontrollable time and space dependent magnetic fields lead to energetic shifts of the internal levels, which result in relative phase shifts destroying the coherence of the system [27].

In this section, we propose a slight modification of the implementation suggested in Sec. III B. This scheme is based on symmetrizing the procedure and avoids the problems mentioned above. Instead of moving the lattices with constant velocity, they oscillate around the central position $x_{0}$ $=0$. In the simplest case, the movement of the lattices is harmonic and may be described as follows:

$$
\begin{gathered}
x^{\prime}(t)=d / 2\left(\cos \omega_{L} t-1\right), \\
x^{\prime \prime}(t)=-d / 2\left(\cos \omega_{L} t-1\right),
\end{gathered}
$$

where $x^{\prime}(t)\left[x^{\prime \prime}(t)\right]$ is the position of the central potential well of lattice 0 [1], respectively. The oscillation frequency $\omega_{L}$ is chosen in such a way that the adiabaticity requirement for the lattice movement-i.e., the atom remains in the mo- 
tional ground state throughout the procedure-is well fulfilled, which leads to oscillation times of the order of $100 \mu \mathrm{s}--1 \mathrm{~ms}$. Note that one may replace the simple harmonic movement of the lattices by more complicated profiles, either specially suited to meet adiabaticity requirements such as the one proposed in Ref. [20], or specially designed in such a way that adiabaticity requirements need not be matched, but are replaced by the weaker condition that the atoms - after moving the lattice by one period - are again in the motional ground state [26]. These specially designed profiles may allow movement times of the order of a few tens of microseconds for a shift of one lattice period. The profiles of Refs. $[20,26]$ need to be adopted in such a way that the lattices oscillate around a central position, which may be accomplished by choosing the original profile until the lattice is displaced by one lattice period and the velocity is zero to a good approximation, and then use the time-inverse profile. In this way, periodic lattice movements are readily achieved. In what follows, we will restrict our discussion to harmonic lattice movements, which is sufficient to illustrate the ideas of the improved implementation of the Hadamard walk. This is realized if, in addition, $\sigma_{x}$ operations ( $\pi$ pulses) are applied at times $t_{n} \equiv n \pi / \omega_{L}, n=1,2,3, \ldots$ to all lattice sites, with the effect $|0\rangle \leftrightarrow|1\rangle$.

Under these conditions, an atom initially in state $|0\rangle$ is trapped in lattice 0 and starts moving to the left together with lattice 0 , where at time $t_{1}$, it is located at position $d$ and the internal state changes to $|1\rangle$ due to the application of $\sigma_{x}$. The particle is, therefore, now trapped in lattice 1, where it moves to the left in the interval $\left(t_{1}, t_{2}\right)$, leaving the trapped atom at position $2 d$ at time $t_{2}$, etc. On one hand, we have that at times $t_{n}$-when manipulations of the internal states of the atoms are performed, the two lattices are on top of each other and the velocity of the atoms is zero, which overcomes the first difficulty mentioned above. One could in principle also stop the lattice movement at these times until manipulation of the internal states is achieved, which allows to drop the requirement that manipulation of the internal states of the atom have to be fast compared to the time scale of the lattice movement. On the other hand, since within a time span of $2 t_{1}$, the atom is in both the states $|0\rangle$ and $|1\rangle$ for time $t_{1}$, relative phase shifts between internal states $|0\rangle$ and $|1\rangle$ become an irrelevant global phase shift. Also fluctuation of magnetic fields become irrelevant, provided the timescale of the fluctuations is much larger than $2 t_{1}$ and the spatial variation is negligible within $2 d$ [28]. These requirements are well fulfilled, e.g., for the typical $50 \mathrm{~Hz}$ background noise and oscillating times $t_{1} \approx 100 \mu \mathrm{s}--1 \mathrm{~ms}$ when using harmonic lattice movements.

The implementation of the Hadamard walk using this setup is straightforward. After the $\sigma_{x}$ operations at times $t_{n}$, the Hadamard operation $H$ [see Eq. (3)] is applied at times $t_{n}$ if $n$ is even, while $H^{\prime}=\sigma_{x} H \sigma_{x}$ is applied at times $t_{n}$ if $n$ is odd. That is, at $t_{0}, H$ is applied, while at $t_{n}, H^{\prime} \sigma_{x}$ $=\sigma_{x} H\left[H \sigma_{x}\right]$ is applied if $n$ is odd (even). The use of the operation $H^{\prime}$ instead of $H$ results from the interchanged role of the internal states $|0\rangle$ and $|1\rangle$ for even (odd) $n$. One readily checks that in this way, after time $t_{n}, n$ steps of the quantum random Hadamard walk are implemented, provided the internal state of the atom is $1 / \sqrt{2}(|0\rangle+i|1\rangle)$. The additional application of $H, H^{\prime}$ does not influence the symmetrization discussed in the previous paragraph. Fluorescence measurements can be performed as described in Sec. III B.

\section{Bounded quantum walks and quantum walk on a circle}

Using above setup, one can also implement an one- or two-side bounded quantum walk on a line $[8,14]$. Such a bounded quantum walk is a simple generalization of its unbounded counterpart and was studied, e.g., in Refs. [8,14]. It has been argued that studying the behavior of such (simple) quantum walks may help to determine the properties of more complicated quantum walks on general graphs, which may offer possible applications in quantum algorithmic design. The bounded quantum walk is such that at certain locations $x_{1}, x_{2}$, barriers are introduced and the quantum walk ends once a particle reaches one of these locations. A one-side bounded quantum walk may, e.g., contain a barrier at $x_{1}$ $=100$, while $x_{2} \rightarrow-\infty$. Such a barrier can in the optical lattice setup, for instance, be implemented by shining a laser at a certain location $x_{1}$, which couples both states $|0\rangle$ and $|1\rangle$ to a fast decaying auxiliary level. In such a way, a (position) measurement of the atom projecting onto $P_{1}$ $=\left|x_{1}\right\rangle_{X}\left\langle x_{1}\right|$, is performed.

A modification of the trapping geometry may also allow for the implementation of a quantum walk on a circle, following the ideas of a recent proposal by Burke et al. [23], using an evanescent field of a linear waveguide and a ring resonator for trapping and guiding atoms (see also Ref. [24]). In this way, the periodic trapping potential can be modified such that lattices sites are located on a circle, forming a regular spaced pattern. Movements of the trapping potentials result in this case in a circular movement of the lattice sites and thus of the trapped particle. By means of Hadamard rotations, together with lattice movements, a quantum walk on a circle could be implemented. Measurements and manipulations of the trapped atom can be performed in the same way as discussed in Secs. III B and III C. Note that in contrast to the quantum walk on the line, the quantum walk on the circle cannot be implemented using existing experimental setups but rather relies on a proposed scheme.

\section{E. Using 2D setup to measure probability distribution of a $1 D$ quantum walk}

In current experiments, two-dimensional (2D) or threedimensional (3D) lattice arrays are used rather than 1D arrays. In this case, four (six) interfering laser beams constitute the two- (three-) dimensional trapping potential. One can make use of such a setup to directly measure the probability distribution of the one-dimensional quantum walk. Consider a two-dimensional lattice, which may be loaded from a Bose-Einstein condensate (BEC) [22]. We assume that the 2D lattice is loaded in such a way that in one dimension, say $x$, only the central lattice site $x_{0}$ is occupied, while in the other dimension, say $y$, all lattice sites are occupied in a regular way with one atom per site. This can be accomplished, for example, by first realizing a Mott transition in a 3D optical lattice from a BEC to a Mott insulator state as in 
recent experiments of Greiner et al. [22], following a theoretical proposal of Ref. [29]. In a second step, one can, e.g., use a gradient magnetic field to selectively address specific lattice layers and to deplete unneeded lattice sites [25]. One may also apply methods similar to the ones used in Ref. [30] to achieve uniform filling factors.

Such a configuration allows for a parallel sampling of one-dimensional quantum walks (in the $x$ direction), by moving the lattices in the $x$ direction only and applying Hadamard rotations to all atoms as described in Secs. III B and III C. Each of the atoms at position $y_{k}=k d$ independently performs a quantum walk. A fluorescence measurement, e.g., a projective picture along the $y$ axis, allows one to directly measure the corresponding probability distribution $p(n)$, provided the number of lattice sites in $y$ direction is big enough. Otherwise, the required number of repetitions of the experiment to determine the probability distribution is decreased.

\section{EXPERIMENTAL IMPERFECTIONS}

Although existing experiments using optical lattice systems offer high accuracy in both coherent storage and manipulation of the atoms, different kinds of errors may influence the ideal evolution. These errors may disturb or even destroy typical features of the quantum walk, such as linear spreading time. In this section, we concentrate mainly on errors in the coherence of the internal states of the atoms. We thereby observe a transition from quantum-mechanical to classical behavior of the walk for increasing errors. We expect these kind of errors to constitute the dominating part in the experimental imperfections. On one hand, errors in lattice movements may lead to motional excitations of the atom. If sufficiently small, these should however, not effect the essential behavior of the system. On the other hand, the internal states of the atom are influenced by the decoherence resulting from, e.g., uncontrollable phase shifts and imperfections in the manipulation by means of laser pulses as well as fluctuations in the trapping potential during lattice shifts. One may distinguish between errors introduced by manipulations of internal state of the atom and errors in the position space of the atom, e.g., introduced by tunneling of atoms between neighboring lattice sites. While the former always keeps the structure of the ideal Hadamard walk that after $n$ time steps, only even (odd) lattice sites are occupied if $n$ is even (odd), respectively, errors in the latter lead to occupations of all lattice sites.

We use two simple models to investigate imperfections in the coherence of the internal state of the atom. We treat decoherence effects and errors in the manipulation of the atom (imperfect Hadamard operations), i.e., operations acting on the total Hilbert space $\mathcal{H}_{I} \otimes \mathcal{H}_{X}$ as $U_{I} \otimes 1_{X}$, in a joint way. In the first model, we assume that the desired manipulation of the internal states of the atom, $U$, is performed with probability $p$ at each time step, while with probability $1-p$, a completely depolarized, random state is produced. The parameter $p$ serves not only as a measure of the accuracy of the operation-where $p=1$ describes perfect operations, while $p=0$ corresponds to a completely random operation-but also includes other decoherence effects due to storage errors or phase fluctuations as well as lattice movements. Such a covariant error model reflects our limited knowledge about the specific type of error that occurred in the system. This error model has also been used in other contexts [31] and is described by the following mapping:

$$
\mathcal{E}(\rho)=p U_{I} \rho U_{I}^{\dagger}+(1-p) 1 / 2 \mathbb{1}_{I} \otimes \operatorname{tr}_{I}(\rho) .
$$

Note that this model is equivalent to a (partially) depolarizing channel, $\mathcal{E}(\rho)=p U_{I} \rho U_{I}^{\dagger}+(1-p) 1 / 4 \sum_{k=0}^{3} \sigma_{k}^{(I)} \rho \sigma_{k}^{(I)}$, where $\sigma_{k}$ are Pauli matrices with $\sigma_{0} \equiv 1$.

The second model only includes phase errors and is motivated by the expectation that phase fluctuation may be the dominating part of errors occurring in optical lattice systems. This error model is described by the following mapping:

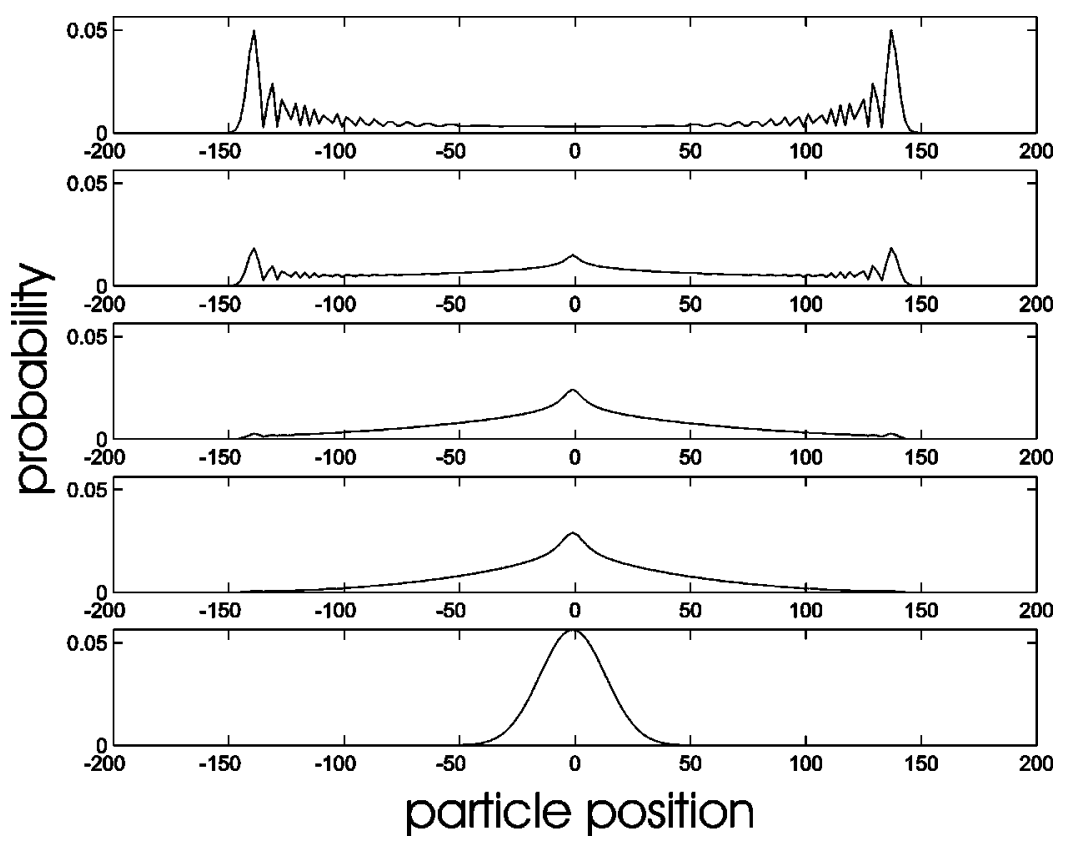

FIG. 1. Probability distribution after $n=200$ steps of quantum walk on a line with imperfect operations using error model 1 [see Eq. (6)]. Error parameter $p=1,0.99,0.97,0.95,0$ from top to bottom. Only even positions are plotted, since odd positions are not occupied. The lowest curve corresponds to the probability distribution of the classical random walk on the line. 


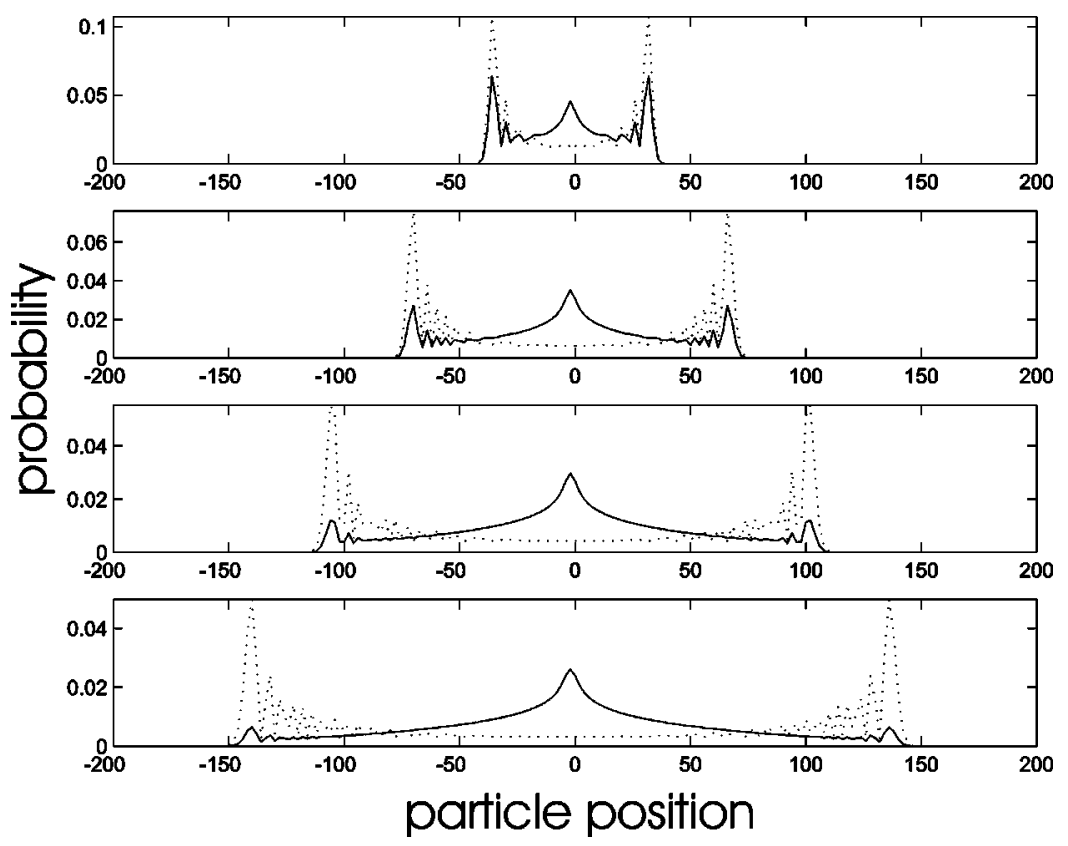

FIG. 2. Probability distribution for ideal quantum walk on a line (dotted line) and quantum walk with imperfect operations using error model 2 [see Eq. (7)] and $p^{\prime}=0.98$ (solid line) after $n$ $=50,100,150,200$ steps (from top to bottom). Only even positions are plotted, since odd positions are not occupied.

$$
\mathcal{E}^{\prime}(\rho)=p^{\prime} U_{I} \rho U_{I}^{\dagger}+\left(1-p^{\prime}\right) U_{I} \sigma_{3}^{(I)} \rho \sigma_{3}^{(I)} U_{I}^{\dagger}
$$

Note that if the optical potentials are not very deep, tunneling between neighboring sites may occur as well. We have used a simple model of incoherent tunneling — affecting only the position of the atom-which is given by the following mapping [32]:

$$
\mathcal{E}^{\prime \prime}(\rho)=q \rho+(1-q) / 2\left(U_{+} \rho U_{+}^{\dagger}+U_{-} \rho U_{-}^{\dagger}\right),
$$

where $U_{ \pm}=1_{I} \otimes \Sigma_{k}|k \pm 1\rangle_{X}\langle k|$ is the unitary shift operator which moves the particle either one position to the left, $U_{-}$ or right, $U_{+}$. That is, with probability $q$ nothing happens, and thus in total, the desired evolution occurs, while with probability $(1-q)$, tunneling of the atom to one of the neighboring lattice sites occurs.

We have performed numerical simulations to investigate the influence of these kinds of errors on the quantum walk on the line, where we first assumed that errors affect only the internal state of the atom. Figure 1 is based on error model 1 and shows the probability distribution after $n=200$ steps of the quantum walk for different error parameters. Note that for completely random operations, i.e., parameter $p=0$, the particle performs exactly a classical random walk. This can easily be understood by observing that an internal state $1 / 21=1 / 2(|0\rangle\langle 0|+| 1\rangle\langle 1|)$, when applying the controlledshift operation (i.e., the lattice movement), has the effect that the particle moves with probability $1 / 2$ to the left, while with probability $1 / 2$, it moves to the right. In contrast to the quan-
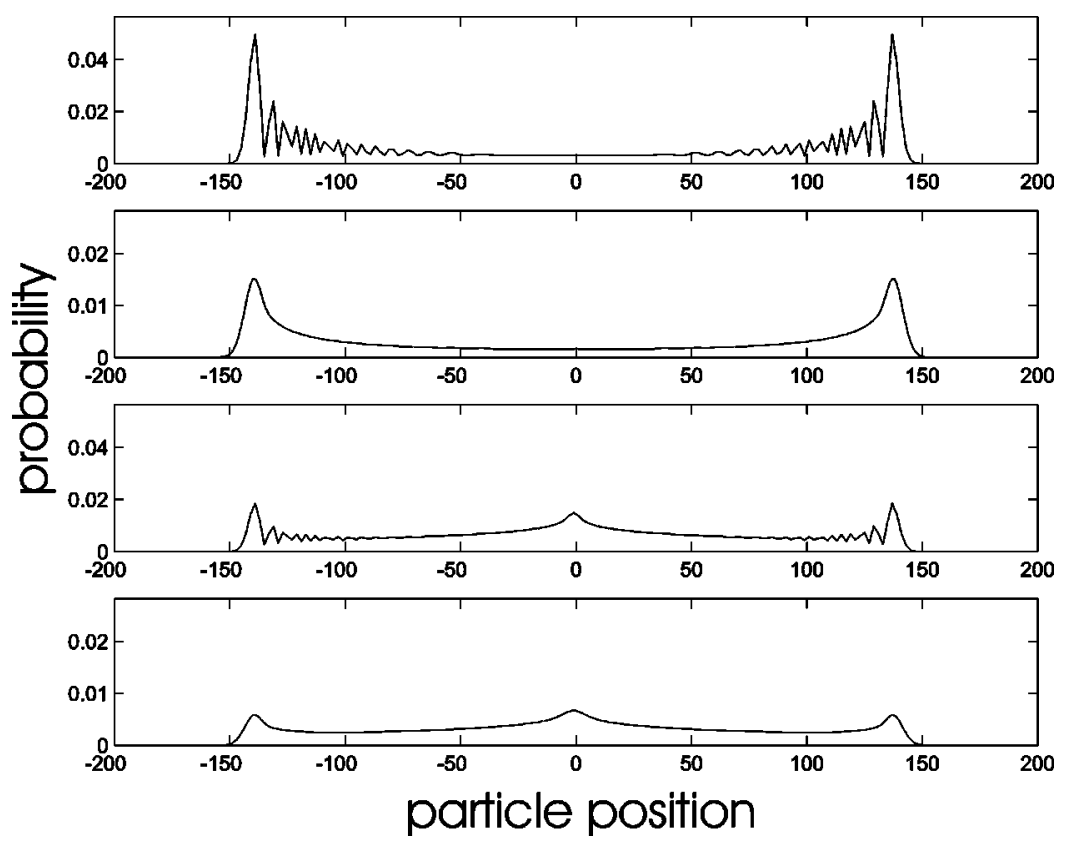

FIG. 3. Probability distribution after $n=200$ steps of quantum walk on a line with imperfect operations using error model 1 [see Eq. (6)] affecting the internal state of the atom and errors due to tunneling described by Eq. (8). Error parameters $\quad(p ; q)=(1 ; 1),(1 ; 0.95),(0.99 ; 1)$, $(0.99 ; 0.95)$ from top to bottom. For $q=1$, only even positions are plotted because odd positions are not occupied. For $q<1$, even and odd positions are occupied and plotted, which explains the lower occupation of specific sites as compared to $q=1$. 


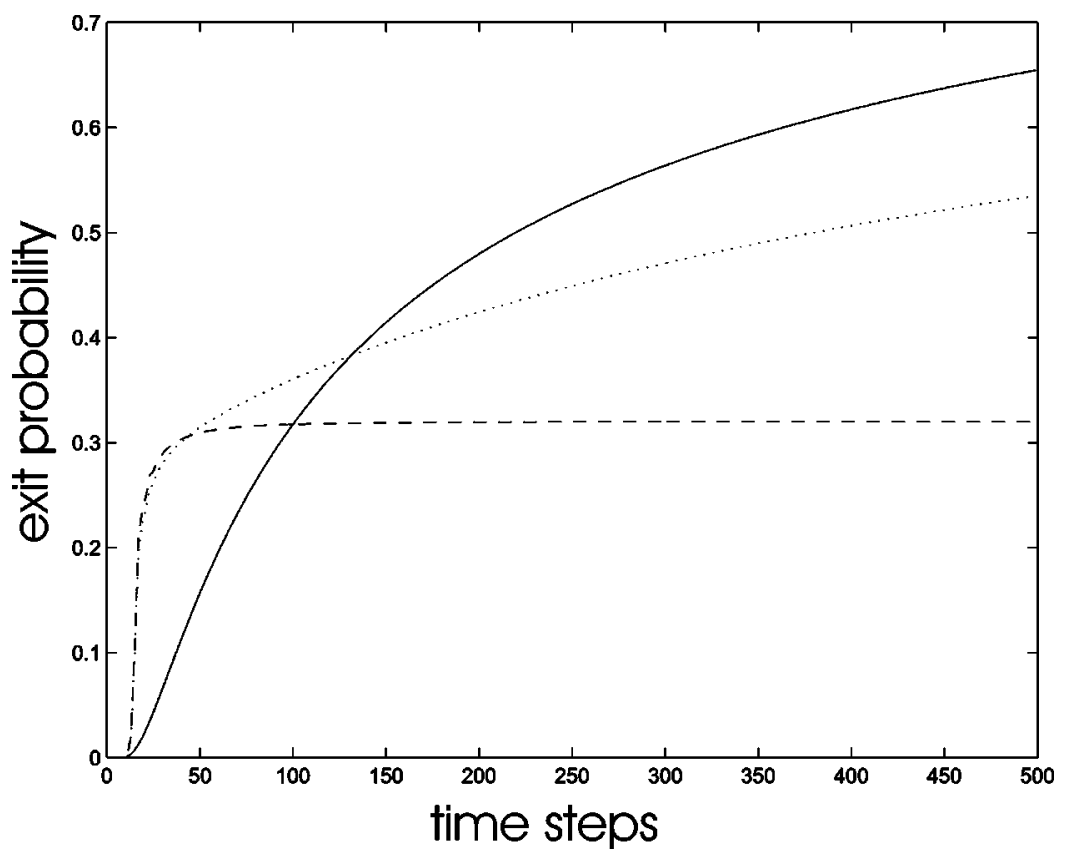

FIG. 4. Bounded quantum walk with barrier at position $x=-10$. Probability that atom was observed at the barrier plotted as a function of the number of steps for classical random walk (solid line), ideal quantum walk (dashed line), and quantum walk with imperfections in the manipulation of the internal state of the atom (dotted line), using error model 1 and $p=0.99$ [see Eq. (6)].

tum walk, the resulting state is an incoherent superposition of the two possible states, which can be described classically and thus no interference effects (as in the quantum walk) occur. The internal state plays the role of a classical coin. One observes from Fig. 1 that with increasing errors (decreasing parameter $p$ ), the probability distribution changes from the quantum-mechanical one to the classical one. Even for errors of the order of several percent, typical quantummechanical features of the probability distribution after a few hundred steps are clearly visible, in particular occupation in the interval $(\sqrt{n}, n / \sqrt{2})$ can be observed. A similar simulation was performed using error model 2 [Eq. (7)]. The observed behavior of the system under this kind of error is very similar to the one shown in Fig. 1 using error model 1. Figure 2 shows the probability distribution after different number of steps of the ideal (imperfect) quantum walk assuming only phase errors.

In the following, we assume both internal and external errors (tunneling), described by Eqs. (6) and (8), respectively. As shown in Fig. 3, the essential effect of incoherent tunneling is that the probability distribution is smeared out.

We have also considered a one-side bounded quantum walk. While the exit probability of the classically bounded random walk approaches unity, this is not the case for the quantum walk (see e.g., Ref. [14]). Figure 4-which shows the probability to observe the atom at the barrier at position $x=-10$ as a function of the number of steps-confirms this behavior for the bounded classical random walk and the ideal bounded quantum walk, and also illustrates the influence of errors on the exit probability.

Although no reliable estimates for the parameters $p$ including all possible imperfections and decoherence effects are available, errors of the order of several percents are still tolerable to observe a clear quantum behavior of the quantum walk, even after a few hundred steps. This seems to be experimentally achievable. In turn, the distribution measured in the experiment can be used to determine the degree of co- herence of the system, in particular the quality of the implemented operations. This may also serve as a test on the suitability of optical lattice systems to perform general purpose quantum computation, following the proposals of Refs. [20,33].

\section{SUMMARY AND CONCLUSIONS}

We have proposed to use neutral atoms trapped in optical lattices to implement quantum walks on the line and on the circle. The quantum walk is performed in position space by periodically shifting the lattices and manipulating the internal states of the atom(s) by homogeneous laser pulses. Readout of the resulting probability distribution is performed via fluorescence measurements. Due to long lifetimes of the trapped atoms and efficient manipulation techniques, experimental realizability is expected with present-day technology. We have also investigated the influence of decoherence and imperfections in manipulation of internal state of the atoms and showed a transition taking place from the ideal quantum walk to the classical random walk for increasing errors. Errors of the order of percent seem tolerable to still observe a clear quantum behavior of the walk after a few hundred steps.

\section{ACKNOWLEDGMENTS}

We would like to thank Immanuel Bloch and Markus Greiner for valuable discussions. H.-J.B. would like to thank Ignacio Cirac and Peter Zoller for useful comments. This work was financially supported by the European Union through Grant No. HPMF-CT-2001-01209 (W.D.), IST-199913021, and the Deutsche Forschungsgemeinschaft. V.K. was funded by the UK Engineering and Physical Sciences Research Council Grant No. GR/N2507701. 
[1] P.W. Shor, in Proceedings of the 35th Annual Symposium on Foundations of Computer Science (IEEE Computer Society Press, Los Alamitos, CA, 1994), p. 124.

[2] L.K. Grover, Phys. Rev. Lett. 79, 325 (1997).

[3] M.N. Barber and B.W. Ninham, Random and Restricted Walks: Theory and Applications (Gordon and Breach, New York, 1970).

[4] M. Jerrum, A. Sinclair, and E. Vigoda, Proc. 33rd STOC 712 (2001); R. Motwani and P. Raghavan, Randomized Algorithms (Cambridge University Press, Cambridge, UK, 1995); U. Schöning, in Proceedings of the 40th Annual Symposium on Foundations of Computer Science (IEEE, New York, 1999), pp. 17-19.

[5] Y. Aharanov, L. Davidovich, and N. Zagury, Phys. Rev. A 48, 1687 (1993).

[6] E. Farhi and S. Gutmann, Phys. Rev. A 58, 915 (1998).

[7] D. Aharonov, A. Ambainis, J. Kempe, and U. Vazirani, in Proceedings of the ACM Symposium on Theory of Computation (STOC '01) 2001 (ACM Press, New York, 2001), pp. 50-59; see also e-print quant-ph/0012090, pp. 50-59.

[8] A. Ambianis, E. Bach, A. Nayak, A. Vishwanath, and J. Watrous, in Proceedings of the 30th Annual ACM Symposium on Theorie of Computing (Association for Computing Machinery, New York, 2001), pp. 37-49.

[9] T.D. Mackay, S.D. Bartlett, L.T. Stephenson, and B.C. Sanders, J. Phys. A 35, 2745 (2002).

[10] A.M. Childs, E. Farhi, and S. Gutmann, e-print quant-ph/0103020; C. Moore and A. Russell, e-print quant-ph/0104137; J. Du, H. Li, X. Xu, J. Wu, X. Zhou, and R. Han, e-print quant-ph/0203120; N. Konno, T. Namiki, and T. Soshi, e-print quant-ph/0205065.

[11] J. Kempe, e-print quant-ph/0205083.

[12] T. Yamasaki, H. Kobayashi, and H. Imai, e-print quant-ph/0205045.

[13] B.C. Travaglione and G.J. Milburn, Phys. Rev. A 65, 032310 (2002).

[14] E. Bach, S. Coppersmith, M.P. Goldschen, R. Joynt, and J. Watrous, e-print quant-ph/0207008.

[15] B.C. Sanders, S.D. Bartlett, B. Tregenna, and P.L. Knight, e-print quant-ph/0207028.

[16] D.A. Meyer, Phys. Lett. A 223, 337 (1996).

[17] This choice of input state ensures a symmetric behavior of the quantum walk, while other input states may lead to asymmetric probability distributions. Throughout this paper, we will consider input states that lead to symmetric probability distributions, our proposal and analysis is, however, not restricted to such input states.

[18] I. Deutsch and P.S. Jessen, Phys. Rev. A 57, 1972 (1998).

[19] R. Grimm, M. Weidemüller, and Yu.B. Ovchinnikov, Adv. At., Mol., Opt. Phys. 42, 95 (2000).
[20] D. Jaksch, H.-J. Briegel, J.I. Cirac, C.W. Gardiner, and P. Zoller, Phys. Rev. Lett. 82, 1975 (1999); H.-J. Briegel, T. Calarco, D. Jaksch, J.I. Cirac, C.W. Gardiner, and P. Zoller, J. Mod. Opt. 47, 415 (2000).

[21] G.K. Brennen, C.M. Caves, P.S. Jessen, and I.H. Deutsch, Phys. Rev. Lett. 82, 1060 (1999).

[22] M. Greiner, I. Bloch, O. Mandel, T.W. Hänsch, and T. Esslinger, Phys. Rev. Lett. 87, 160405 (2001); M. Greiner, O. Mandel, T. Esslinger, T.W. Hänsch, and I. Bloch, Nature (London) 415, 39 (2002).

[23] J.P. Burke, Jr., S.-T. Chu, G.W. Bryant, C.J. Williams, and P.S. Julienne, Phys. Rev. A 65, 043411 (2002).

[24] C. Williams, talk delivered at the DFG meeting on Quantum Information Processing, Bad Honnef, Germany, 2002 (unpublished), see also website http://physics.nist.gov/Divisions/ Div842/div842.html

[25] I. Bloch (private communication).

[26] D. Polivaev, Master thesis, Ludwig Maximians Universität München, Germany, 2000; D. Polivaev and H.-J. Briegel (unpublished).

[27] Depending on the energy $E_{k} \propto F|B(x, t)|$ of the hyperfine structure levels $F=1(|0\rangle)$ and $F=2(|1\rangle)$, we have that $|k\rangle$ $\rightarrow e^{-i \phi_{k}}|k\rangle$, where $\phi_{k} \propto E_{k} t$.

[28] Using that $B(x, t) \approx B_{0}$ within $2 t_{1}$ and two lattice sites, we have that after a complete cycle (including also a final $\sigma_{x}$ operation), i.e., $2 t_{1},|k\rangle \rightarrow e^{-i\left(\phi_{0}+\phi_{1}\right)}|k\rangle$, which implies that relative phases became a global, irrelevant phase. For example, a particle in state $|0\rangle$ picks up a phase shift $\phi_{0} t_{1}$ within the first time interval $\left(0, t_{1}\right)$, while a phase shift $\phi_{1} t_{1}$ is picked up in the second time interval $\left(t_{1}, t_{2}\right)$.

[29] D. Jaksch, C. Bruder, J.I. Cirac, C.W. Gardiner, and P. Zoller, Phys. Rev. Lett. 81, 3108 (1998).

[30] M.T. DePue, C. McCormick, S.L. Winoto, S. Oliver, and D.S. Weiss, Phys. Rev. Lett. 82, 2262 (1999).

[31] H.-J. Briegel, W. Dür, J.I. Cirac, and P. Zoller, Phys. Rev. Lett. 81, 5932 (1998); W. Dür, H.-J. Briegel, J.I. Cirac, and P. Zoller, Phys. Rev. A 59, 169 (1999).

[32] We have restricted our investigation to incoherent tunneling effects. One may also investigate the influence of coherent tunneling on the quantum walk. In this case, tunneling serves as an additional source of interference, which may affect the interference constituting the basis of the quantum walk. A detailed analysis of such influence is, however, beyond the scope of this paper.

[33] R. Raussendorf and H.-J. Briegel, Phys. Rev. Lett. 86, 5188 (2001).

[34] A.M. Childs, R. Cleve, E. Deotto, E. Farhi, S. Gutmann, and D.A. Spielman, e-print quant-ph/0209131.

[35] N. Shenvi, J. Kempe, and K.B. Whaley, e-print quant-ph/0210064. 